\title{
Giant cell tumor of the tendon sheath: A rare case in the left knee of a 15-year-old boy
}

\author{
CHENGTAO SUN, WEI SHENG, HUIMING YU and JUNQING HAN \\ Tumor Research and Therapy Center, Provincial Hospital affiliated to Shandong University, \\ Shandong University, Jinan, Shandong 250021, P.R. China
}

Received September 19, 2011; Accepted December 19, 2011

DOI: $10.3892 / \mathrm{ol} .2012 .555$

\begin{abstract}
Localized forms of giant cell tumors are defined as giant cell tumors of the tendon sheath (GCTTS). GCTTS arises from the synovium of a joint, bursa or tendon sheath, and $85 \%$ of the tumors occur in the fingers. GCTTS in the knee is extremely rare. We report an unusual case of a 15 -year-old boy who presented with an occult growing swelling and a 2-month history of infra-patellar pain in the left knee. Magnetic resonance imaging (MRI) demonstrated a well-circumscribed soft tissue mass in the infra-patellar fat pad posterior to the patella tendon. Excision biopsy was performed by surgical removal. Histopathological examination revealed that it was GCTTS. During the follow-up period, his recovery was propitious and there was no recurrence. Owing to its few and non-specific symptoms, and local recurrence varying from 9 to $44 \%$, its proper, early diagnosis and appropriate treatment is necessary. The purpose for which we report the case is to emphasize the possibility of GCTTS where there is a mass with non-specific symptoms such as infra-patellar pain of the knee, and to avoid misdiagnosis where possible.
\end{abstract}

\section{Introduction}

Giant cell tumor of the tendon sheath (GCTTS) is a benign lesion of uncertain etiology that involves inflammation, trauma, toxin, allergy, clonal chromosomal abnormalities and aneuploidy (1-2). Jaffe et al originally described the condition in 1941 (3). Approximately 85\% of GCTTS occurs in the fingers, while $12 \%$ of tumors are located in the knee, elbow, hip and ankle (4). GCTTS may occur at any age but most typically occurs between the ages of 30 and 50, with a 2:1 female predominance (5). GCTTS in the knee is extremely rare,

Correspondence to: Professor Junqing Han, Tumor Research and Therapy Center, Provincial Hospital Affiliated to Shandong University, 324 Jingwu Weiqi Road, Jinan, Shandong 250021, P.R. China

E-mail: hanjq1960@126.com

Key words: giant cell tumor, knee, tendon sheath, diagnosis, histopathology, magnetic resonance imaging particularly in a 15-year-old boy. Most often, GCTTS is small (average tumor size, $2.0 \mathrm{~cm}$ ) (5), although the mass in this case, measuring $3.8 \times 2.0 \times 1.7 \mathrm{~cm}$ was much larger than average. There are few reports in the literature of cases occurring in males, in younger age groups, tumor located in the knee and of greater tumor size. Antecedent trauma has been reported, but only $15 \%$ of cases have a definite history of trauma (6). Our patient reported a trauma while playing basketball at school 6 months previously. Treatments consist of careful local excision, particularly microscopic excision, and postoperative radiotherapy. GCTTS may recur following excision.

One of the most important clinical features is that the symptoms of GCTTS are non-specific. We report a valuable case that was not initially regarded as GCTTS. Following excision biopsy, the diagnosis of GCTTS was made. We believe that this study may assist clinicians in making a proper diagnosis and administering correct treatment.

\section{Case report}

The research findings are presented with the consent of the boy's parents and ethics committee approval. A 15-year-old boy presented with an occult growing swelling and a 2-month history of pain in the left knee at the Provincial Hospital Affiliated to Shandong University, China. Strenuous exercise aggravated the pain, which was felt at the infra-patellar region of the left knee. The skin temperature of the left knee was higher than the right one. The patient reported a trauma when playing basketball at school 6 months previously. Physical examination revealed local tenderness of the fibular and tibial collateral ligament. The range of motion was limited. Hyperextension and hyperflexion tests of the left knee were positive. There were no constitutional symptoms such as night sweats, fever or weight loss. The patient had previously been healthy, and no enlarged inguinal lymph nodes were detected. Analysis of the patient's blood was normal.

Plain radiography and magnetic resonance imaging (MRI) were obtained. Plain anteroposterior and lateral radiographs revealed no bone erosion of the left knee joint (Fig. 1). MRI demonstrated an encapsulated soft tissue lump in the infra-patellar fat pad, posterior to the patella tendon (Fig. 2). Initially, we regarded the neoplasm as an intra-articular lipoma, synovial sarcoma or fibroma of the tendon. Excision biopsy was performed by surgical removal, and an irregular shaped mass 

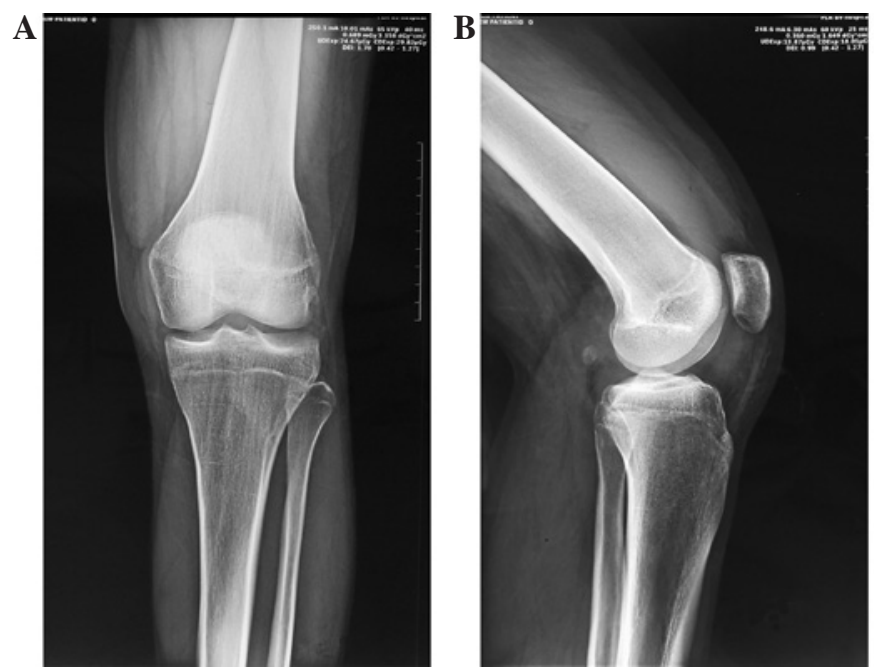

Figure 1. Plain radiography of the left knee. (A) Anteroposterior and (B) lateral $\mathrm{X}$-rays show no bone erosion.

A

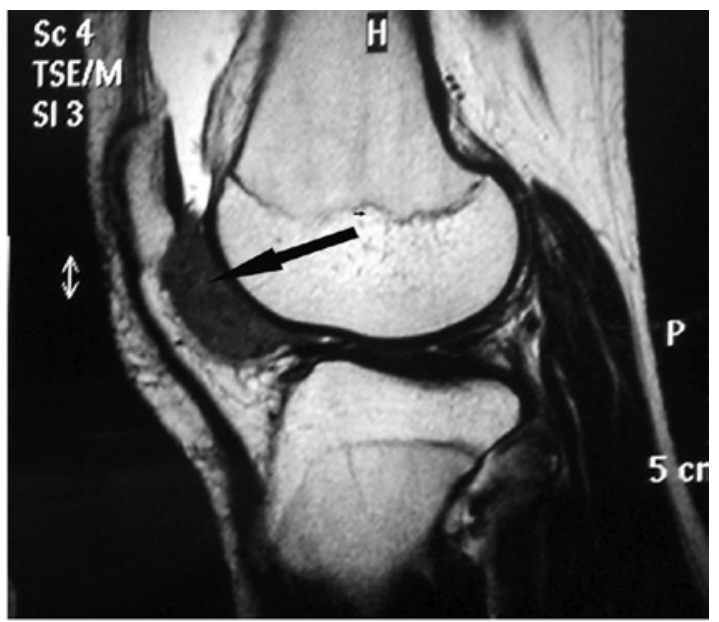

B

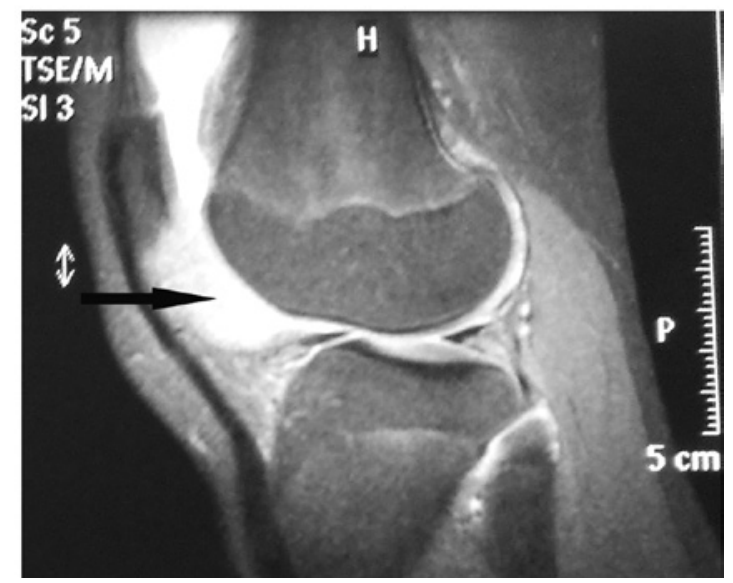

Figure 2. Magnetic resonance imaging (MRI) of the left knee (A) T1-weighted and (B) T2-weighted MR images show a well-circumscribed soft tissue mass (arrows) in the infra-patellar fat pad posterior to the patella tendon.

measuring $3.8 \times 2.0 \times 1.7 \mathrm{~cm}$ was removed. Histopathological examination of the specimen revealed prominent mononuclear cells, accompanied by a variable number of foamy histiocytes, hemosiderin-laden macrophages and multinucleated giant cells (Fig. 3). No mitotic activity or malignant feature was reported. A diagnosis of GCTTS was finally made.

The recovery following surgery was propitious. There was no local tenderness of the left knee and the skin temperature of the knee was normal. The patient had full range of motion of the knee joint and returned to school life with no difficulty. During the 5 month follow-up period there was no evidence of local tumor recurrence.

\section{Discussion}

The diffuse and localized extra-articular forms of pigmented villonodular synovitis (PVNS) are presently referred to as diffuse-type giant cell tumor and giant cell tumor of the tendon sheath (GCTTS), respectively (7), and were originally alluded to by Jaffe et al in 1941 (3). Most localized giant cell tumors are small (average tumor size, $2.0 \mathrm{~cm}$ ) (5), although lesions of greater size may be found in large joints such as the knee and elbow. Tumors are well-circumscribed and typically lobulated, and white to gray in color, with yellowish and brown areas. Most diffuse-type giant cell tumors are infiltrative and grow as diffuse, expansile sheets. These entities are now classified as 'fibriohistiocytic tumors' in the World Health Organization system of classification of bone and soft tissue tumors (4). GCTTS occurs in the digits of the hands and feet more frequently than in the large joints of the knee, elbow and ankle (8); $85 \%$ of GCTTS is observed in fingers, while $12 \%$ is observed in large joints (4). GCTTS in the knee is rare.

The etiology of GCTTS remains uncertain. A variable number of factors have been described as possible causes of GCTTS. These include inflammation, trauma, toxin, allergy, clonal chromosomal abnormalities and aneuploidy (1-2). GCTTS was initially regarded as an inflammatory disease; however, the finding of aneuploidy in certain cases and the demonstration of clonal chromosomal abnormalities strongly support a neoplastic origin (4).

The diagnosis of GCTTS may be difficult. Patients typically present with a painless mass in the digits or large joints. The lesions are usually well-circumscribed and localized, and infrequently erode or infiltrate the nearby bone (6). In large joints, the symptoms are non-specific and few, thus the diagnosis is difficult to make. The soft tissue mass grows and expands into areas of least resistance, such as the tendon sheath (8). Plain radiographs are usually not helpful in the diagnosis of the disease. Bone erosions or soft tissue swelling is occasionally found in plain radiographs.

Magnetic resonance imaging (MRI) is an effective and highly sensitive tool for diagnosis. Typically, MRI features T1- (T1WI) and T2-weighted (T2WI) images, which may present with a homogeneous low signal intensity in GCTTS. The reason for this is that tumors contain dense collagen and hemosiderin-laden macrophages (9).

Treatments consist of careful and complete local excision and postoperative radiotherapy. Surgical removal is the standard treatment of GCTTS. Adequate initial local excision may effectively limit the risk of local recurrence (10). Recently, microscopic excision has been prescribed to prevent recurrence. Ikeda et al (11) treated 18 patients with microscopic excision, and of the 18 patients which only one case recurred. The reason for this recurrence was that the microscope 
A

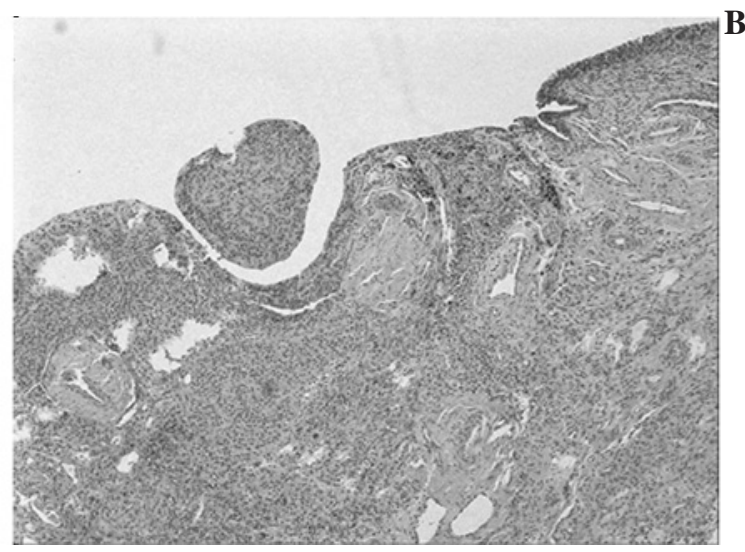

B

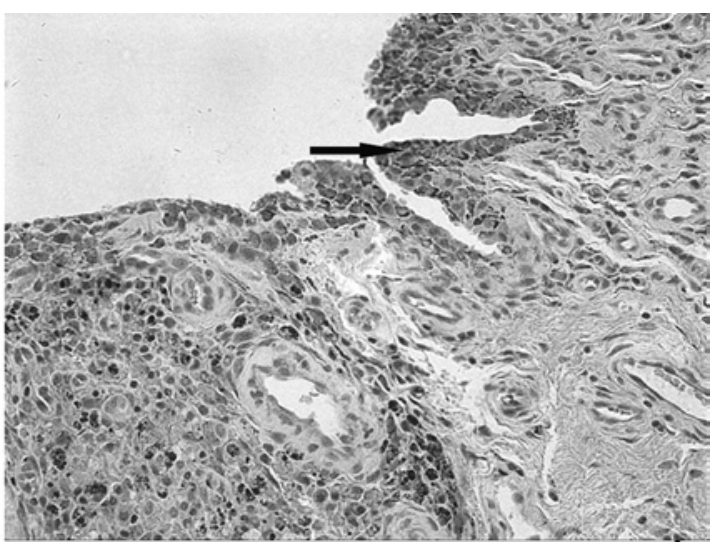

Figure 3. (A) Low-power view of GCTTS reveals a prominent number of mononuclear cells with scattered multinucleated giant cells (hematoxylin and eosin, original magnification x40). (B) High-power view of GCTTS demonstrating a variable number of hemosiderin granules (arrow) located in multinucleated giant cells and sheets of mononuclear cells (hematoxylin and eosin, original magnification x100). GCTTS, giant cell tumor of the tendon sheath.

excision was not used in this diffuse-type patient. As an adjuvant therapy, postoperative radiotherapy had been indicated to prevent recurrence following surgery. Kotwal et al (12) published a prospective study in which the recurrence rate was only $4 \%$. This rate was much lower than previously reported incidences of between 25 and $45 \%$.

Although GCTTS is a benign type of tumor, it has a high incidence of recurrence. Recurrence following resection has been a consistent problem, and occurs in 9 to $44 \%$ of cases (13). Lowyck and De Smet (13) retrospectively reviewed 49 patients and reported the rate of recurrence to be $16 \%$.

In conclusion, the concurrence of GCTTS occurring in a younger male, occurring in the knee and being of greater than average size is unique to our patient, making this an exceptional case to those reported in previous literature. It is extremely difficult to make a proper diagnosis during the earlier stages of GCTTS. As the condition always presents with a painful lump, it should be considered as a differential diagnosis if the mass is found in the knee, particularly in younger males and when the tumor is of greater size. MRI may be useful for diagnosis when the symptoms of GCTTS are non-specific and few. To make a definite diagnosis, histopathological examination is required. If a possible diagnosis of GCTTS is suspected, excision biopsy should be carried out as early as possible in the same manner that the present case was treated. Finally, the purpose for which we report this case is to emphasize the possibility of GCTTS, even when the painless or painful mass accompanied with non-specific symptoms are found in the knee of a younger patient, and to avoid misdiagnosis wherever possible.

\section{References}

1. Reilly KE, Stern PJ and Dale JA: Recurrent giant cell tumors of the tendon sheath. J Hand Surg 24: 1298-1302, 1999.

2. Abdul-Karim FW, el-Naggar AK, Joyce MJ, Makley JT and Carter JR: Diffuse and localized tenosynovial giant cell tumor and pigmented villonodular synovitis: a clinicopathologic and flow cytometric DNA analysis. Hum Pathol 23: 729-735, 1992.
3. Jaffe HL, Lichtenstein L and Sutro CJ: Pigmented villonodular synovitis, bursitis and tenosynovitis. Arch Pathol 31: 731-765, 1941.

4. Fletcher CDM, Krishnan Unni K and Mertens F (eds): Giant cell tumour of tendon sheath In: World Health Organization Classification of Tumors, Pathology and Genetics of Tumors of Soft Tissue and Bone. IARC Press, Lyon, pp110-111, 2002.

5. Ushijima M, Hashimoto H, Tsuneyoshi M and Enjoji M: Giant cell tumor of the tendon sheath (nodular tenosynovitis): a study of 207 cases to compare the large joint group with the common digit group. Cancer 57: 875-884, 1986.

6. Uriburu IJ and Levy VD: Intraosseous growth of giant cell tumors of the tendon sheath (localized nodular tenosynovitis) of the digits: report of 15 cases. J Hand Surg 23: 732-736, 1998.

7. Sanghvi DA, Purandare NC, Jambhekar NA, Agarwal MG and Agarwal A: Diffuse-type giant cell tumor of the subcutaneous thigh. Skeletal Radiol 36: 327-330, 2007.

8. Relwani J, Factor D, Khan F and Dutta A: Giant cell tumor of the patellar tendon sheath-an unusual cause of anterior knee pain: a case report. Knee 10: 145-148, 2003.

9. Cheng XG, You YH, Liu W, Zhao T and Qu H: MRI features of pigmented villonodular synovitis (PVNS). Clin Rheumatol 23: 31-34, 2004.

10. Monaghan H, Salter DM and Al-Nafussi A: Giant cell tumour of tendon sheath (localized nodular tenosynovitis): clinicopathological features of 71 cases. J Clin Pathol 54: 404-407, 2001.

11. Ikeda K, Osamura N and Tomita K: Giant cell tumor in the tendon sheath of the hand: importance of the type of lesion. Scand J Plast Reconstr Surg Hand Surg 41: 138-142, 2007.

12. Kotwal PP, Gupta V and Malhotra R: Giant-cell tumor of the tendon sheath: is radiotherapy indicated to prevent recurrence after surgery? J Bone Joint Surg Br 82: 571-573, 2000.

13. Lowyck H and De Smet L: Recurrence rate of giant cell tumors of the tendon sheath. Eur J Plast Surg 28: 385-388, 2006. 